ORIGINAL ARTICLE

\title{
Effect of Metformin Therapy on Clinical and Hormonal Indices of Patients with Polycystic Ovary Syndrome
}

\author{
SUMAIRA INAYAT ${ }^{1}$, HINA FAZAL KHATTAK ${ }^{2}$, MOBASHARA GHULAM MUHAMMAD ${ }^{3}$, KOUSAR ROBEEN ${ }^{4}$, AFSHAN \\ INAYAT ${ }^{5}$, FATIMA $^{6}$ \\ ${ }^{1}$ Senior Registrar, Obstetrics \& Gynaecology Department, Northwest General Hospital and Research Center, Peshawar. \\ ${ }^{2}$ Women Medical Officer, Mian Rashid Hussain Shaheed Memorial Hospital, Pabbi \\ ${ }^{3}$ Senior Registrar Obstetrics \& Gynaecology Department, Mohtarma Benazir Bhutto Shaheed Medical College, Mirpur AJK \\ ${ }^{4}$ Senior Registrar Obstetrics \& Gynaecology Department, Mohammad College of Medicine, Muhammad Teaching Hospital, Peshawar \\ ${ }^{5}$ Medical Officer, Category D Badaber Hospital, Peshawar \\ ${ }^{6}$ Junior Registrar, Obstetrics \& Gynaecology Department, Mardan Medical Complex Mardan \\ Corresponding author: Dr Sumaira Inayat, Email: sumairainayat@hotmail.com, Cell\#: +92 3339402029
}

\begin{abstract}
Objective: The main objective of this study is to determine the efficacy of metformin therapy on clinical and hormonal indices of patients with polycystic ovary syndrome.

Study Design: Randomized control trial

Place and Duration: Study was conducted at Obstetrics \& Gynaecology department of Northwest General Hospital and Research Center, Peshawar and Mian Rashid Hussain Shaheed Memorial Hospital, Pabbi for duration of nine months from March 2020 to November 2020.

Methods: Total 100 patients of polycystic ovary syndrome were presented in this study. Patients were aged between 18 to 45years. Patients detailed demographics including age, body mass index and socioeconomicstatus were recorded after taking informed written consent. Patients were divided into 2-groups, group I had 50 patients and received metformin $(500 \mathrm{mg})$ three times a day and group II had 50 patients and received pioglitazone $(30 \mathrm{mg}$ ) three times a day for 3months. Clinical (body weight, blood pressure (BP), and body mass index) and indices fasting blood sugar (FBS), serum triglyceride (TG), cholesterol, low-density lipoprotein, highdensity lipoprotein, insulin, testosterone, and dehydroepiandrosterone (DHEA) were measured before and after therapy. Complete data was analyzed by SPSS 22.0 version.

Results: Mean age of the patients in group I was $29.18 \pm 2.25$ years with mean BMI $26.14 \pm 8.86 \mathrm{~kg} / \mathrm{m}^{2}$ and in group II mean age was $29.8 \pm 2.52$ years with mean BMI $27.64 \pm 7.68 \mathrm{~kg} / \mathrm{m}^{2}$.Significantly decrease in blood

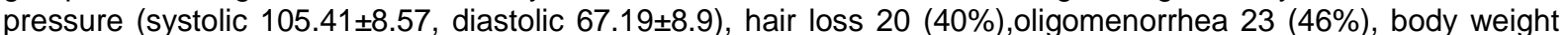
$74.45 \pm 9.72$, waist circumference and dehydroepiandrosterone (DHEA) $2.35 \pm 0.67$ and as compared to group II. Only triglycerides gave results of reduction in group II 115.39 \pm 64.11 . Among both groups serum insulin, acne, menstrual disturbance and fasting blood sugar were controlled after treatment.

Conclusion: We concluded in this study that use of metformin in patients with polycystic ovary syndrome resulted in to decrease clinical body weight, blood pressure (BP), body mass index and hormonal indices with reduction of serum insulin, acne, menstrual disturbance and fasting blood sugar but pioglitazone was an alternative effective and reliable method in PCOS patients.
\end{abstract}

Keywords: Polycystic ovary syndrome, Insulin resistance, Metformin, Pioglitazone

\section{INTRODUCTION}

Polycystic Ovary Syndrome (PCOS) in women of reproductive age is a prevalent endocrine gynecological disease[1]. Excessive androgen, Chronic anovulation continuous ovulation, infertility and metabolic problems are characteristic with PCOS[2]. During the reproductive time, the morbidity rate is $6-15 \%$ among females, and the cause is still not fully apparent. Approximately $50 \%$ of PCOS patients are overweight or non overweight[3], with comprehensive clinical and epidemiological evidence. PCOS-induced overweight women are more serious than non-overweight patients with endocrine and metabolism[4]. Surveys have indicated that overweight strength improves the secretion of insulin, but impairs the liver, skeletal muscle, and fat metabolism of insulin separation. Overweight can also lead to lipodystrophy and insulin resistance by lowering the lipid droplet protein expression in adipocytes in addition to poor adipocyte insulin reception $[5,6]$. Karimy et al [7] and Heshmati et al. [8] imply that insulin and serum metabolism and aberrant lipoprotein metabolism are typically present in people with polycystic ovarian syndrome. Studies showed that PCOS women are at a higher risk for type 2 diabetes, high blood pressure, overweight pulmonary illness, cardiovascular illness and metabolic syndrome [9].

Palombaetal. has noted that the lowered fertility rate that PCOS patients demonstrate cannot be attributed alone to chronic anovulation, [10] and other causes of poor fertility, affecting the quality and/or abnormality of the oocyte, are likely to cause other variables. Moreover, the risk of miscarriage, gestational diabetes, hypertension or preeclampsia seems to be more exposed than the usual populations. [11]

It has been recently clarified that PCOS traits can be influenced by both genetic and environmental factors.[10,12] In fact, there is a statistically significant rise in PCOS in patients' first-degree families compared to several groups, which shows a potential hereditary susceptibility of the condition [12]. Indeed, several investigations have shown that various genes are involved in the PCOS pathogenesis and that each gene interferes according to the pathophysiological mechanism. 
Environmental factors such as particular lifestyle, dietary kinds, housing situations and also the impact of intrauterine growth could play an important role for such genetic predispositions[12]. Actually, in intrauterine growth may contribute to hyperandrogen and ovarian dysfunction later in life if exposure is defined by excess androgen levels.[12]

Pioglitazone's side effects include moderate to severeoedema, weight gain, small hemoglobin and hematocrit decreases, myalgia, and transient elevations in liver enzymes.[13] Metformin is generally well-tolerated, but occasionally causes gastrointestinal adverse effects, including vomiting. [14, 15]

\section{MATERIAL AND METHODS}

This randomized control trial was conducted at Obstetrics \& Gynaecology department of Northwest General Hospital and Research Center, Peshawar and Mian Rashid Hussain Shaheed Memorial Hospital, Pabbi for duration of nine months from March 2020 to November 2020and included 100 patients. Patients details demographics were recorded after taking written consent. Patients with cardiac renal disease, liver failure and other severe medical illness were excluded from this study.

Patients were aged between 18-45 years. Patients details demographics age, body mass index and socio economicstatus were recorded after taking informed written consent. Patients were divided into 2-groups, group I had 50 patients and received metformin $(500 \mathrm{mg})$ three times a day and group II had 50 patients and received pioglitazone (30 mg) three times a day for 3months. Clinical (body weight, blood pressure (BP), and body mass index) and hormonal indices fasting blood sugar (FBS), serum triglyceride (TG), cholesterol, low-density lipoprotein, highdensity lipoprotein, insulin, testosterone, and dehydroepiandrosterone (DHEA) were measured before and after therapy. Chi square test was used to analyzed the data.

\section{RESULTS}

Mean age of the patients in group I was $29.18 \pm 2.25$ years with mean BMI $26.14 \pm 8.86 \mathrm{~kg} / \mathrm{m}^{2}$ and in group II mean age was $29.8 \pm 2.52$ years with mean BMI $27.64 \pm 7.68$ $\mathrm{kg} / \mathrm{m}^{2}$. According to socio-economic status $60(60 \%)$ females were from middle class and 40 (40\%) were from upper class. $56(56 \%)$ cases were from urban area and 44 (44\%) patients were from rural area. 66 (66\%) patients were literate and 34 (34\%) cases were illiterate. (table 1)

Table 1: Baseline details demographics of enrolled cases

\begin{tabular}{|l|l|l|}
\hline Variables & Group I (n=50) & Group II (n=50) \\
\hline Mean Age & $29.18 \pm 2.25$ & $29.8 \pm 2.52$ \\
\hline Mean BMI & $26.14 \pm 8.86$ & $27.64 \pm 7.68$ \\
\hline Socio-economic status & $30(30 \%)$ & $30(30 \%)$ \\
\hline Middle class & $20(20 \%)$ & $20(20 \%)$ \\
\hline Upper class & $28(28 \%)$ & $28(28 \%)$ \\
\hline Residential status & $22(22 \%)$ \\
\hline Urban & $22(22 \%)$ & $33(33 \%)$ \\
\hline Rural & $33(33 \%)$ & $17(17 \%)$ \\
\hline Education Status & $17(17 \%)$ & \\
\hline Literate &
\end{tabular}

Significantly decrease in hair loss 20 (40\%), oligomenorrhea 23 (46\%), amenorrhea 1 (2\%), polymenorhea 0 and acne 22 (44\%) in group I as compared to the group of pioglitazone in which loss hair found 32 (64\%), oligomenorrhea $40(50 \%)$, amenorrhea 3 (6\%), polymenorhea 2(4\%) and acne 23 (46\%). (table 2$)$

Table 2: Qualitative outcomes among both groups after treatment

\begin{tabular}{|l|l|l|}
\hline Variables & Group I & Group II \\
\hline Hair Loss & $20(40 \%)$ & $32(64 \%)$ \\
\hline Oligomenorrhea & $23(46 \%)$ & $25(50 \%)$ \\
\hline Amenorrhea & $1(2 \%)$ & $3(6 \%)$ \\
\hline polymenorhea & 0 & $2(4 \%)$ \\
\hline Acne & $22(44 \%)$ & $23(46 \%)$ \\
\hline
\end{tabular}

After treatment to both groups we found significantly decrease in blood pressure (systolic 105.41 \pm 8.57 , diastolic $67.19 \pm 8.9$ ), body weight $74.45 \pm 9.72$, waist circumference $86.19 \pm 7.5$ and mean BMI $25.34 \pm 6.18$ in metformin group as compared to pioglitazone group in which blood pressure (systolic 114.14 \pm 8.57 , diastolic $69.91 \pm 8.9$ ), body weight $76.54 \pm 9.27$, waist circumference $89.17 \pm 4.8$ and mean BMI $26.43 \pm 5.1 \mathrm{~kg} / \mathrm{m}^{2}$. (table 3)

Endocrine indices fasting blood sugar (FBS), cholesterol, low-density lipoprotein, high-density lipoprotein, insulin, testosterone, and dehydroepiandrosterone were significantly decreases among group I but only serum triglyceride (TG) was decreases in group II as compared to group I. (table 4)

Table 3: Clinical outcomes among both groups

\begin{tabular}{|l|l|l|}
\hline Variables & Group I & Group II \\
\hline Systolic & $105.41 \pm 8.57$ & $114.14 \pm 8.57$ \\
\hline Diastolic & $67.19 \pm 8.9$ & $69.91 \pm 8.9$ \\
\hline Body weight & $74.45 \pm 9.72$ & $76.54 \pm 9.27$ \\
\hline Waist circumstance & $86.19 \pm 7.5$ & $89.17 \pm 4.8$ \\
\hline BMI & $25.34 \pm 6.18$ & $26.43 \pm 5.1$ \\
\hline
\end{tabular}

\begin{tabular}{|l|l|l|}
\hline Variables & Group I & Group II \\
\hline fasting blood sugar & $83.46 \pm 7.98$ & $84.52 \pm 5.54$ \\
\hline low-density lipoprotein & $87.64 \pm 15.13$ & $94.12 \pm 62.04$ \\
\hline high-density lipoprotein & $47.97 \pm 6.78$ & $49.17 \pm 14.34$ \\
\hline insulin & $16 \pm 5.79$ & $19.37 \pm 8.41$ \\
\hline testosterone & $1.95 \pm 2.64$ & $2.02 \pm 1.07$ \\
\hline dehydroepiandrosterone & $2.35 \pm 0.67$ & $2.99 \pm 3.68$ \\
\hline serum triglyceride (TG) & $115.39 \pm 64.11$ & $117.39 \pm 65.06$ \\
\hline
\end{tabular}

\section{DISCUSSION}

In PCOS, a prevalent women's endocrine condition, hyperinsulinemia is a key pathogenic component. Therefore, therapies that lower insulin resistance are of potential clinical relevance. [15]The effectiveness of pioglitazone versus metformin in PCOS treatment is compared in this study. The methods of treatment have been compared with metabolic, endocrine and clinical outcomes. While for several characteristics substantial group differences were discovered, no regime in this investigation using the here mentioned treatment schemes proved better than the other. In our study Mean age of the patients in group I was $29.18 \pm 2.25$ years with mean BMI $26.14 \pm 8.86 \mathrm{~kg} / \mathrm{m}^{2}$ and in group II mean age was $29.8 \pm$ 2.52 years with mean BMl $27.64 \pm 7.68 \mathrm{~kg} / \mathrm{m}^{2}$. According to socio-economic status $60(60 \%)$ females were from middle 
class and $40(40 \%)$ were from upper class. 56 (56\%) cases were from urban area and $44(44 \%)$ patients were from rural area. 66 (66\%) patients were literate and 34 (34\%) cases were illiterate.[25]

In present study total 100 patients were presented and divided equally into 2-groups. Group I receivedmetformin $(500 \mathrm{mg}$ ) three times a day and group II had 50 patients and received pioglitazone $(30 \mathrm{mg})$ three times a day for 3months and follow up was taken for 8 months. The study has found a reduction inhyperglycemia Hyperanemia and Hyperandrogenemia and ultimately a remedying of the menstrual cycle, hair loss and acne in females with PCOS, using metformin and Pioglitazone. Following treatment in both groups, acne is clearly improved. Improvement of menstruation problems was also important in both groups. The effects of both metformin and pioglitazone in menstruation and acne treatment have been agreed in most research. [18,20]

In this study BMI was not decreased significantly in both groups $(25.34 \pm 6.18,26.43 \pm 5.1)$, which contradictions $\mathrm{Li}$ et al results, there was no significant decline in BMI. [16] Velazquez et al. observed that waist circumference reductions occurred after therapy with metformin. [17] Our Endocrine Fasting Blood Sugar (FBS) study reported significant decreases in group I, with only serum triglyceride (TG) deteriorations in group II in comparison with group I, cholesterol, low density lipoprotein, high density of lipoprotein and insulin, testosterone and dehydroepiandrosterone. Sarkozy and others. [18] . However, Ortega-Gonzalez et al. have observed that once pioglitazone is administered, the waist circumference is substantially raised. $[19,20]$ The circumferences of tails $>35$ inches among women show aberrant hormonal and metabolic functions, which are one of the cardiovascular disease risk factors. [21] FBS and insulin fasting fell substantially in both groups with therapy. The combination of metformin and pioglitazone decreases insulin, glucose and insulin resistance in patients not responding to metformin by themselves in Glueck et al. [22] After therapy with metformin, blood lipid levels did not alter. Only TG levels have fallen dramatically in the pioglitazone group, and the amount of this reduction.

After therapy with metformin, blood lipid levels have not changed. Only TG levels have fallen dramatically in the pioglitazone group, and the amount of this reduction was minimum. In the study of Naka et al, metformin therapy lowers LDL levels, but pioglitazone therapy increases LDL and chol and HDL. [20] Furthermore, a great deal of research demonstrated that metformin not only reduces weight and metabolic diseases, but also corrects menstrual patterns, restores ovulations and even makes designs possible. [23,24] In addition, the specific treatment effects of metformin on metabolic markers have not been investigated by prior systematic reviews in PCOS overweight women. Through quantitative synthesis, we observed that metformin seems to be effective as a medicine which regulates the metabolism of women with overweight PCOS. It can lower BMI and WC and lower testosterone, FSH, LH and LDL cholesterol.

\section{CONCLUSION}

We concluded in this study that metformin is an effective method to decrease clinical body weight, blood pressure
(BP), body mass index and hormonal indices with reduction of serum insulin, acne, menstrual disturbance and fasting blood sugar but pioglitazone was an alternative effective and reliable method in PCOS patients.

\section{REFERENCE}

1. R. S. Legro, S. A. Arslanian, D. A. Ehrmann et al., "Diagnosis and treatment of polycystic ovary syndrome: an endocrine society clinical practice guideline," The Journal of Clinical Endocrinology \& Metabolism, vol. 98, no. 12, pp. 4565-4592, 2013.

2. R. J. Norman, D. Dewailly, R. S. Legro, and T. E. Hickey, "Polycystic ovary syndrome," The Lancet, vol. 370, no. 9588, pp. 685-697, 2007.

3. L. Mu, Y. Zhao, R. Li, Y. Lai, and J. Qiao, "Metabolic characteristics of normal weight central obesity phenotype polycystic ovary syndrome women: a large-scale national epidemiological survey," Reproductive Biomedicine Online, vol. 37, no. 4, pp. 498-504, 2018.

4. S. Arya, K. R. Hansen, and R. A. Wild, "Metformin rosiglitazone, or both for obese women with polycystic ovary syndrome?" Fertility and Sterility, vol. 113, no. 1, pp. 87-88, 2020.

5. B. Shi, D. Feng, M. Sagnelli et al., "Fructose levels are elevated in women with polycystic ovary syndrome with obesity and hyperinsulinemia," Human Reproduction, vol. 35, pp. 187-194, 2020

6. M. P. Czech, "Insulin action and resistance in obesity and type 2 diabetes," Nature Medicine, vol. 23, no. 7, pp. 804814, 2017.

7. E. Karimi, J. Heshmati, N. Shirzad et al., "The effect of synbiotics supplementation on anthropometric indicators and lipid profiles in women with polycystic ovary syndrome: a randomized controlled trial," Lipids in Health and Disease, vol. 19, p. 60, 2020.

8. J. Heshmati, F. Farsi, S. Yosaee et al., "The effects of probiotics or synbiotics supplementation in women with polycystic ovarian syndrome: a systematic review and metaanalysis of randomized clinical trials," Probiotics and Antimicrobial Proteins, vol. 11, no. 4, pp. 1236-1247, 2019.

9. N. M. Rashad, Y. S. Saraya, S. A. Afifi, A. E. Ali, and R. M. Al-sayed, "Impact of weight loss on plasma ghrelin level, clinical, and metabolic features of obese women with or without polycystic ovary syndrome," Middle East Fertility Society Journal, vol. 24, 2019.

10. Palomba, S, Falbo, A, Zullo, F, Orio, F: Evidence-based and potential benefits of metformin in the polycycstic ovary syndrome: a comprehensive review. Endocr. Rev. 30,150 (2009)

11. Palomba, S, Falbo, A, Orio, F, Zullo, F: Insulin sensitizing agents and reproductive function in polycystic ovary syndrome patients. Curr. Opin. Obstet. Gynecol. 20, 364373 (2008).

12. Deligeoroglou, E, Kouskouti, C, Christopoulos, P: The role of genes in the polycystic ovary syndrome: predisposition and mechanism. Gynecol. Elndocrinol. 25, 603-609 (2009).

13. Vinaixa M, Rodriguez MA, Samino S, Díaz M, Beltran A, Mallol R, et al. Metabolomics reveals reduction of metabolic oxidation in women with polycystic ovary syndrome after pioglitazone-flutamide-metformin polytherapy. PLoS One 2011;6:e29052

14. Roe A, Hillman J, Butts S, Smith M, Rader D, Playford M, et al. Decreased cholesterol efflux capacity and atherogenic lipid profile in young women with PCOS. J Clin Endocrinol Metab 2014;99:E841-7.

15. Haydardedeoglu B, Simsek E, Kilicdag EB, Bagis T. Metabolic and endocrine effects of metformin and metformin plus cyclic medroxyprogesterone acetate in women with 
polycystic ovary syndrome. Int J GynaecolObstet2009;105:32-5

16. Li XJ, Yu YX, Liu CQ, Zhang W, Zhang HJ, Yan B, et al Metformin vs thiazolidinediones for treatment of clinical, hormonal and metabolic characteristics of polycystic ovary syndrome: A meta-analysis. Clin Endocrinol (Oxf) 2011;74:332-9

17. Velazquez EM, Mendoza S, Hamer T, Sosa F, Glueck CJ. Metformin therapy in polycystic ovary syndrome reduces hyperinsulinemia insulin resistance, hyperandrogenemia, and systolic blood pressure, while facilitating normal menses and pregnancy. Metabolism 1994;43:647-54

18. Romualdi D, Guido M, Ciampelli M, Giuliani M, Leoni F, Perri $C$, et al. Selective effects of pioglitazone on insulin and androgen abnormalities in normo-and hyperinsulinaemic obese patients with polycystic ovary syndrome. Hum Reprod2003;18:1210-8

19. Ortega-González C, Luna $S$, Hernández L, Crespo G, Aguayo $\mathrm{P}$, Arteaga-Troncoso G, et al. Responses of serum androgen and insulin resistance to metformin and pioglitazone in obese, insulin-resistant women with polycystic ovary syndrome. J Clin Endocrinol Metab2005;90:1360-5

20. Naka KK, Kalantaridou SN, Kravariti M, Bechlioulis A, Kazakos N, Calis KA, et al. Effect of the insulin sensitizers metformin and pioglitazone on endothelial function in young women with polycystic ovary syndrome: A prospective randomized study. FertilSteril2011;95:203-9

21. Wild RA, Carmina E, Diamanti-Kandarakis E, Dokras A, Escobar-Morreale HF, Futterweit W, et al. Assessment of cardiovascular risk and prevention of cardiovascular disease in women with the polycystic ovary syndrome: A consensus statement by the Androgen Excess and Polycystic Ovary Syndrome (AE-PCOS) Society. J Clin Endocrinol Metab2010;95:2038-49

22. Glueck CJ, Moreira A, Goldenberg N, Sieve L, Wang P. Pioglitazone and metformin in obese women with polycystic ovary syndrome not optimally responsive to metformin. Hum Reprod2003;18:1618-25

23. E. Fraison, L. Moran, S. Bilal, C. Ee, C. Venetis, and M Costello, "Metformin versus the combined oral contraceptive pill for hirsutism, acne, and menstrual pattern in polycystic ovary syndrome," Human Reproduction, vol. 34, p. 458, 2019

24. E. M. Bordewijk, M. Nahuis, M. F. Costello et al., "Metformin during ovulation induction with gonadotrophins followed by timed intercourse or intrauterine insemination for subfertility associated with polycystic ovary syndrome," Cochrane Database of Systematic Reviews, vol. 1, no. 1, 2017

25. Shahebrahimi K, Jalilian N, Bazgir N, Rezaei M. Comparison clinical and metabolic effects of metformin and pioglitazone in polycystic ovary syndrome. Indian J EndocrMetab2016;20:805-9. 